ESJ Natural/Life/Medical Sciences

\title{
Utilisation des lambeaux dans les pertes de substances maxillo-faciales: à propos de 32 cas au Niger
}

\author{
Kadre A.K.O. \\ Mayaou A-K. \\ Service de Stomatologie et Chirurgie Maxillo-Faciale, \\ hôpital général de référence-Niamey, Niger \\ Djafarou $B$. \\ Illé $S$. \\ Service d'ORL et Chirurgie Cervico-Faciale, \\ hôpital général de référence, Niger \\ Moustapha $\boldsymbol{H}$. \\ Service de Chirurgie Pédiatrique, hôpital Amirou Diallo-Niamey, Niger \\ Salami A. \\ Romaric B. \\ Service de Stomatologie et Chirurgie Maxillo-faciale, \\ CHU de Treichville-Abidjan, Côte d'Ivoire \\ N'guessan $Z$. \\ Service de Stomatologie et Chirurgie Maxillo-faciale, \\ CHU de Bouaké, Côte d'Ivoire \\ Timi N. \\ Service d'ORL et Chirurgie Cervico-Faciale, \\ hôpital national de Niamey, Niger
}

Doi:10.19044/esj.2021.v17n17p306

Submitted: 06 April 2021

Accepted: 11 May 2021

Published: 31 May 2021
Copyright 2021 Author(s)

Under Creative Commons BY-NC-ND

4.0 OPEN ACCESS

Cite As:

Kadre A.K.O., Mayaou A-K., Djafarou B., Illé S., Moustapha H., Salami A., Romaric B., N'guessan Z. \& Timi N. (2021). Utilisation des lambeaux dans les pertes de substances maxillo-faciales: à propos de 32 cas au Niger. European Scientific Journal, ESJ, 17(17), 306. https://doi.org/10.19044/esj.2021.v17n17p306

\section{Résumé}

Diverses lésions peuvent rompre l'harmonie anatomique, esthétique et fonctionnelle de la face, parmi lesquelles les pertes de substances (PDS) qui peuvent être d'origine traumatique, infectieuse ou tumorale. L'objectif de ce 
travail est de décrire les aspects épidémiologiques, cliniques et thérapeutiques des patients ayant bénéficiés de prise en charge par lambeaux dans les pertes de substances (PDS) maxillo-faciales. Il s'agit d'une étude rétrospective (du 1er janvier 2016 au 15 juillet 2019) et prospective (du 16 juillet 2019 au 31 octobre 2020), soit une période de 4 ans 10 mois (58mois) réalisée dans les services de Stomatologie et Chirurgie Maxillo-Faciale de l'hôpital national et l'hôpital général de référence de Niamey. Pendant la durée de l'étude, 32 patients de tout âge et des deux sexes ont été enregistré dans le service. La moyenne d'âge était de 34 ans avec des âges inférieur et supérieur de 02 ans et 65 ans. Le sexe féminin était prédominant de $59 \%$ des cas. la tranche d'âge de 31-45 ans est la plus touchée avec 31,25\% des cas. L'étiologie tumorale était la plus fréquente dans $43,75 \%$ suivi des (PDS) post- traumatique dans $34,38 \%$ des cas. la région parotidienne représentait la partie la plus atteinte avec $21,88 \%$ des cas. Les lambeaux régionaux et ceux à distance étaient les plus utilisés dans 35,29\% des cas chacun, et les locaux dans $29,42 \%$. Les nécroses partielles étaient les complications les plus fréquentes soit 15,63\% des cas. Les PDS maxillo-faciale sont souvent responsables de certains préjudice fonctionnel et esthétique qui peuvent altérer la personnalité. Ces PDS font appel à des techniques de reconstruction par lambeaux locorégionaux et à distance avec des indications bien précises.

Mots clés : Lambeau, perte de substances, maxillo-faciale, chirurgie, Niger 


\title{
Use of Flap in Maxillofacial Loss of Substances: About 32 Cases in Niger
}

\author{
Kadre A.K.O. \\ Mayaou A-K. \\ Service de Stomatologie et Chirurgie Maxillo-Faciale, \\ hôpital général de référence-Niamey, Niger \\ Djafarou $B$. \\ Illé $S$. \\ Service d'ORL et Chirurgie Cervico-Faciale, \\ hôpital général de référence, Niger \\ Moustapha $\boldsymbol{H}$.
}

Service de Chirurgie Pédiatrique, hôpital Amirou Diallo-Niamey, Niger

Salami $A$.

Romaric $B$.

Service de Stomatologie et Chirurgie Maxillo-faciale,

CHU de Treichville-Abidjan, Côte d'Ivoire

N'guessan $Z$.

Service de Stomatologie et Chirurgie Maxillo-faciale,

CHU de Bouaké, Côte d'Ivoire

Timi N.

Service d'ORL et Chirurgie Cervico-Faciale, hôpital national de Niamey, Niger

\begin{abstract}
Various lesions can disrupt the anatomical, aesthetic and functional harmony of the face, including loss of substances that can be of traumatic, infectious or tumor origin. The objective of this work is to describe the epidemiological, clinical and therapeutic aspects of patients who have benefited from flap management in maxillofacial loss of substances. This is a retrospective (from January 1, 2016 to July 15, 2019) and prospective (from July 16, 2019 to October 31, 2020), i.e. 4 years 10 months (58 months) carried out in the departments of Stomatology and Maxillofacial surgery at the national hospital and the general reference hospital of Niamey. During the study period, 32 patients of all ages and two sexes were registered in the department. The average age was 34 years with lower and upper ages of 02 years and 65 years. The female sex was predominant in $59 \%$ of cases. the $31-$ 45 age group is the most affected with $31.25 \%$ of cases. Tumor etiology was the most frequent in $43.75 \%$ followed by post-traumatic (PDS) in $34.38 \%$ of cases. the parotid region represented the most affected part with $21.88 \%$ of cases. Regional and remote flaps were used the most in $35.29 \%$ of cases each,
\end{abstract}


and local ones in $29.42 \%$. Partial necrosis was the most frequent complication, i.e. $15.63 \%$ of cases. Maxillofacial loss of substance is often responsible for certain functional and aesthetic damage that can alter the personality. These losses of substance use locoregional and remote flap reconstruction techniques with very precise indications.

Keywords: Flap, loss of substance, maxillofacial, surgery, Niger

\section{Introduction}

La face, vitrine de la personnalité, est au premier plan dans les relations avec l'environnement, aussi bien sur les plans fonctionnel, psychoaffectif, qu'esthétique. Son altération peut avoir des retentissements fonctionnels et psycho-affectifs. Diverses lésions peuvent rompre l'harmonie anatomique, esthétique et fonctionnelle de la face, parmi lesquelles les pertes de substances (PDS) qui peuvent être d'origine traumatique, infectieuse ou tumorale. Seules les petites PDS dans cette région sont possibles pour la fermeture primaire et en général celles de taille moyenne et grande ou complexe nécessitent une reconstruction (Deganello A, 2015). Les lambeaux sont une alternative permettant la prise en charge de ces pertes de substance. Plusieurs types de lambeaux peuvent être utilisés : les lambeaux locaux, régionaux, et à distance. Les PDS de la portion céphalique du corps humain ont toujours suscité des questionnements avec crainte et mysticisme dans la société compte tenu de la défiguration. Il est impératif de reconstruire les fonctions altérées et l'esthétique en vue d'une réinsertion sociale. L'objectif de ce travail est de décrire les aspects épidémiologiques, cliniques et thérapeutiques des patients ayant bénéficiés de reconstruction par lambeaux dans PDS maxillo-faciales.

\section{Patients et méthode}

Cette étude a été réalisée dans les services de Stomatologie et Chirurgie Maxillo-Faciale de l'hôpital national et l'hôpital général de référence de Niamey. Il s'agit d'une étude rétrospective (du 1er Janvier 2016 au 15 Juillet 2019) et prospective (du 16 Juillet 2019 au 31 Octobre 2020), soit une période de 4 ans 10mois (58mois). Pendant la durée de l'étude, 32 patients de tout âge et des deux sexes ont été enregistré dans le service de stomatologie et de chirurgie maxillo-faciale. Les critères d'inclusion étaient les patients ayant bénéficié des lambeaux suite à une perte de substance maxillo-faciale. Les Critère de non inclusion étaient les patients présentant des pertes de substances maxillo-faciales associées à des lésions d'autres parties du corps et ceux ayant bénéficié d'une prise en charge par lambeau non à destination cervico-faciale. Ce travail a été réalisé par le même chirurgien avec un faible plateau technique et une équipe non permanente et non initié à la chirurgie 
plastique et reconstructrice de la tête et du cou. Les variables étudiées étaient épidémiologique (âge, sexe, étiologie), clinique, et thérapeutique.

\section{Résultats}

La moyenne d'âge était de 34 ans avec des âges inférieur et supérieur de 02 ans et 65 ans. Le sexe féminin était prédominant dans notre étude soit $59 \%$ des cas ( $n=19$; sexe masculin $41 \%$ avec $n=13$ ), avec un sex ratio de 0,68 .

Tableau 1: répartition des patients par tranche d'âge de PDS.

\begin{tabular}{lcc}
\hline Age (années) & Effectif & Pourcentage \\
\hline $0-15$ & 5 & 15,63 \\
$16-30$ & 9 & 28,13 \\
$31-45$ & 10 & 31,25 \\
$46-60$ & 6 & 18,75 \\
$61-75$ & 2 & 6,25 \\
Total & $\mathbf{3 2}$ & $\mathbf{1 0 0}$
\end{tabular}

La tranche d'âge de 31-45 ans était la plus touchée dans $31,25 \%$ des cas $(n=10)$.

Tableau 2: répartition des patients en fonction de l'étiologie de PDS

\begin{tabular}{lll}
\hline Étiologies & Effectif & Pourcentage \\
\hline Infectieuse (séquelles de noma) & 3 & 9,38 \\
Tumorale & 14 & 43,75 \\
PDS post-traumatiques & 11 & 34,38 \\
Chéloïdes & 2 & 6,25 \\
Bride cicatricielle & 1 & 3,13 \\
Séquelles de & 1 & 3,13 \\
radiothérapie & 32 & 100 \\
Total & & \\
\hline
\end{tabular}

L'étiologie tumorale est la plus fréquente dans $43,75 \%$ suivi des PDS posttraumatique dans $34,38 \%$. 
Tableau 3: répartition des patient en fonction du siège de $P D S$

\begin{tabular}{|c|c|c|}
\hline Siège & Effectif & Pourcentage \\
\hline Ailes narinaires & 3 & 9,38 \\
\hline Lèvre inférieure & 3 & 9,38 \\
\hline Lèvre supérieure & 2 & 6,25 \\
\hline Pavillon de l'oreille & 3 & 9,38 \\
\hline Région parotidienne & 7 & 21,88 \\
\hline Nasolabiale & 2 & 6,25 \\
\hline Front & 2 & 6,25 \\
\hline Joue & 2 & 6,25 \\
\hline Cuir chevelu & 2 & 6,25 \\
\hline Cavité buccale & 3 & 9,38 \\
\hline Région & 1 & 3,13 \\
\hline \multicolumn{3}{|l|}{ mentonnière } \\
\hline Hémiface & 2 & 6,25 \\
\hline Total & 32 & 100 \\
\hline
\end{tabular}

La région parotidienne représente la partie la plus atteinte dans $21,88 \%$ des cas. 
Tableau 4: répartition en fonction du type de Lambeau

\begin{tabular}{|c|c|c|}
\hline Lambeaux & Effectif & Pourcentage \\
\hline Grand pectoral & 2 & 5,13 \\
\hline Grand dorsal & 2 & 5,13 \\
\hline Ginestet & 2 & 5,13 \\
\hline Eslander & 1 & 2,56 \\
\hline Karapandzic & 3 & 7,69 \\
\hline Fibula avec palette cutanée & 1 & 2,56 \\
\hline Frontal & 1 & 2,56 \\
\hline Scalp & 1 & 2,56 \\
\hline Sous-mental & 1 & 2,56 \\
\hline Sterno-cleido-mastoidien & 1 & 2,56 \\
\hline Antébrachial & 1 & 2,56 \\
\hline Temporal & 3 & 7,69 \\
\hline Nasogénien & 7 & 17,95 \\
\hline Mustardé & 3 & 7,69 \\
\hline Webster & 2 & 5,13 \\
\hline Fasciocutané & 7 & 17,95 \\
\hline Cutané & 1 & 2,56 \\
\hline Total & 39 & 100 \\
\hline
\end{tabular}

Les lambeaux nasogénien et fasciocutané sont les plus utilisés dans 17,95\% chacun. Les lambeaux non pédiculés ont été utilisés dans $47,06 \%$ des cas, suivi des pédiculés dans $41,18 \%$ et des libres dans $11,76 \%$. Les lambeaux régionaux ( figure 1) et ceux à distance (figure 2) ont été utilisés dans 35,29\% des cas chacun, et les locaux (figure 3) dans 29,42\%. 


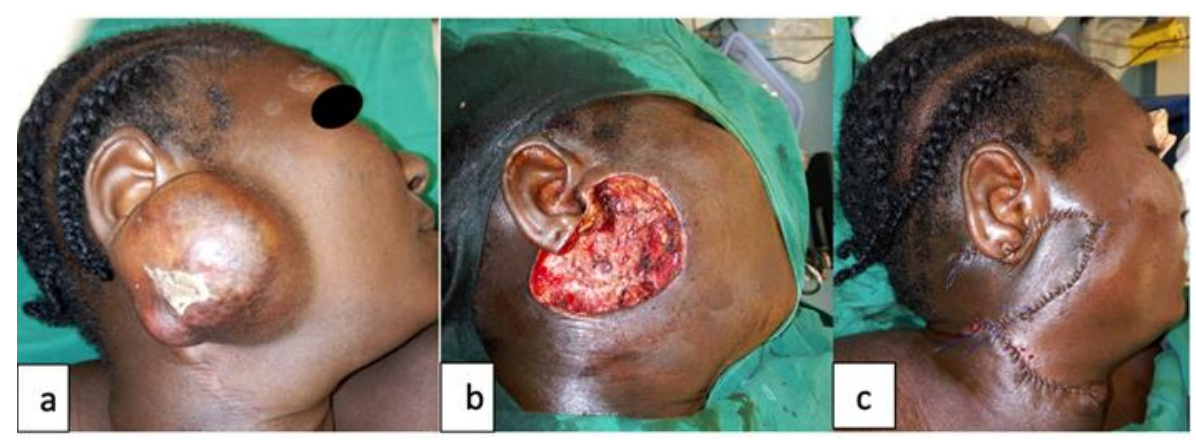

Figure 1: lambeau régional. a : tumeur de la région parotidienne droite; b: PDS cutanée; c : lambeau fascio-cutanée cervico-facial en place

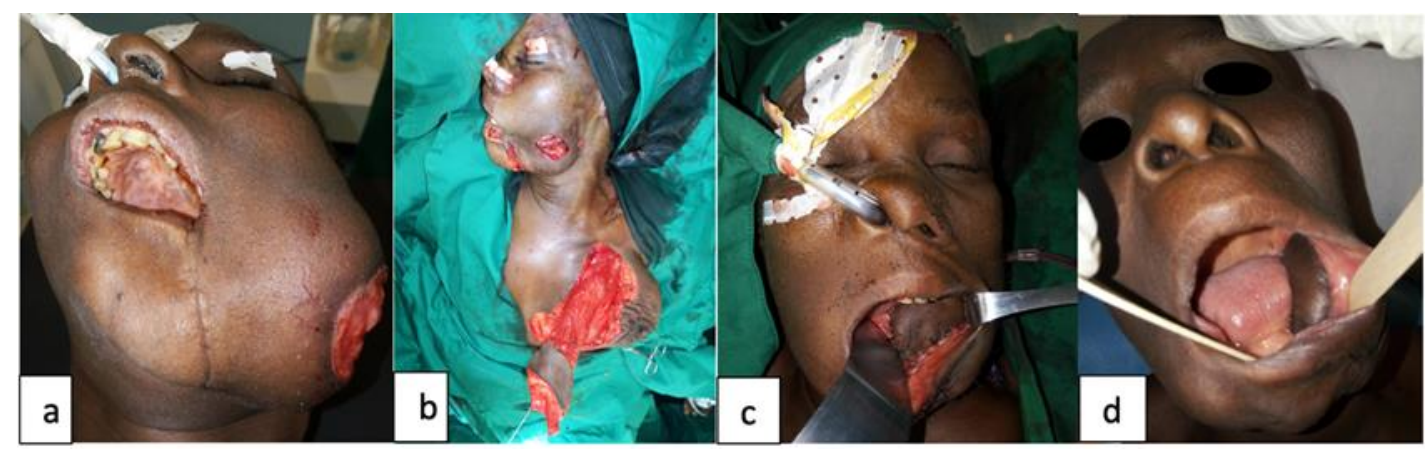

Figure 2: lambeau à distance pédiculé. a: tumeur récidivée génienne gauche; b: exérèse tumorale avec levée de lambeau du grand pectoral avec palette cutanée; c: palette cutanée en bouche; d: vue à 3 mois

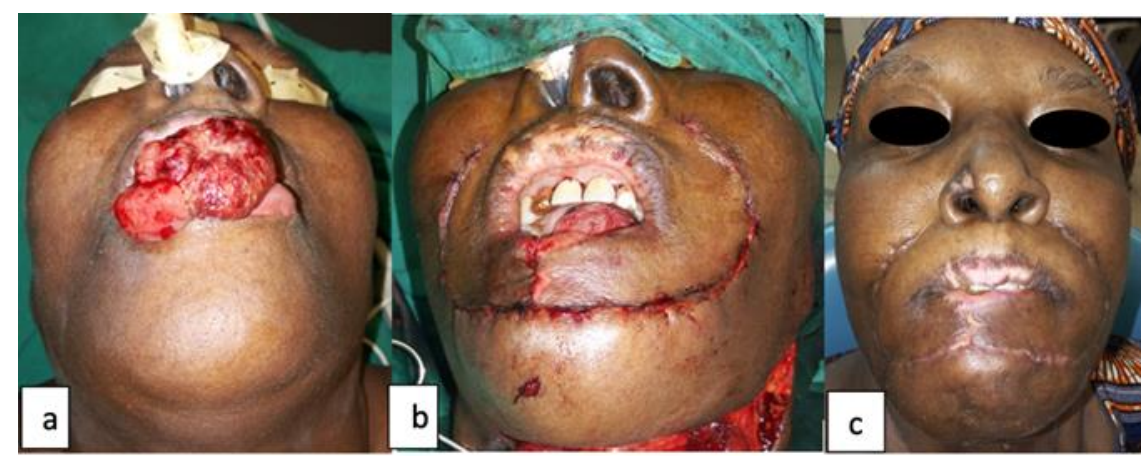

Figure 3: Lambeau local. a : tumeur de la lèvre inférieure; b: lambeau de karapandzick; c: vue à 3 semaines 
Tableau 5: répartition en fonction des complications post-opératoire de la chirurgie des lambeaux

\begin{tabular}{lll}
\hline Complications post opératoires & Effectif (n) & $\begin{array}{l}\text { Pourcentage } \\
(\mathbf{\%})\end{array}$ \\
\hline Hématome du site donneur du grand pectoral & 1 & $\mathbf{3 , 1 3}$ \\
Lymphocèle du site donneur du grand dorsal & 2 & $\mathbf{6 , 2 5}$ \\
Nécrose partielle & 3 & $\mathbf{9 , 3 8}$ \\
Nécrose totale & 2 & $\mathbf{6 , 2 5}$ \\
\hline
\end{tabular}

Les nécroses partielles étaient les complications les plus fréquentes dans $15,63 \%$ des cas.

\section{Discussion}

La face, vitrine de la personnalité et reflet de l'identité, peut être endommagée par divers lésions pouvant occasionner des pertes de substances osseuses ou molles. Si la reconstruction des PDS osseuses fait recours à des greffons osseux vascularisés ou non, celles des parties molles fait recours à l'art plastique des tissus mous, pédiculés ou non. Cette reconstruction vise à restaurer les unités esthétiques de la face altérées, et les fonctions compromises, afin de donner un visage socialement acceptable et une dépendance fonctionnelle. La complexité de l'extrémité céphalique, son importance esthétique et les multiples fonctions qui en dépendent rendent ses reconstructions chirurgicales difficiles. Le souci récent d'une réhabilitation fonctionnelle et cosmétique toujours plus performante a amené de nombreux chirurgiens à rechercher à distance les tissus les plus adaptés aux différentes pertes de substances.

Une gamme assez variée de lambeaux est disponible en Chirurgie reconstructive maxillo- faciale. Ce sont les lambeaux locaux de rotation ou de translation, les lambeaux loco-régionaux, et les lambeaux à distance (Anzouan $\mathrm{K} E$ et al., 2015). Les pertes de substance de la face, quelle que soit l'étiologie, à partir d'une certaine importance, nécessitent une technique plus ou moins simple de reconstruction. La plupart des lambeaux dont l'arc de rotation inclut la région faciale peuvent être envisagés. Le choix de cette reconstruction dépend de la fiabilité, de l'efficacité et des inconvénients associés qui doivent être réduits (Zwetyenga $\mathrm{N}$ et al., 2007). Les objectifs sont de restaurer la forme et la fonction et de minimiser la morbidité du site donneur (Squaquara R et al., 
2010). Étant donné que les individus accordent une grande valeur à l'esthétique du visage, les défauts des tissus mous dans la sphère oro-maxillo-faciale peuvent affecter négativement la perception de la beauté du visage, entraînant une morbidité psychologique significative en plus des problèmes fonctionnels. Les PDS acquises en chirurgie maxillo-faciale sont généralement plus courants et peuvent résulter d'un traumatisme, d'une intervention chirurgicale ou d'infections. Au fil des ans, la reconstruction des défauts des tissus mous orofaciaux a considérablement progressé (Agbara $\mathrm{R}$ et al., 2016). Traditionnellement, l'approche par échelle de reconstruction a été préconisée dans la reconstruction des défauts des tissus mous, ce qui permet une option par étapes, des procédures les plus simples aux procédures les plus complexes. Ces options comprennent la cicatrisation par intention secondaire, la fermeture primaire, la greffe de peau, l'utilisation de lambeaux locorégionaux (avec ou sans expansion tissulaire) et le transfert de lambeau libre (Baliarsing AS et al., 2013). Dans cette étude, le choix des lambeaux a porté sur les lambeaux à distance pédiculé en cas de PDS vaste et pluritissulaire, sur les lambeaux locaux et régionaux au voisinage de la PDS sans aggraver ou engendrer un préjudice fonctionnel ou esthétique orofacial. En cas de lambeau à distance microanastomosés, la chirurgie est longue surtout avec un seul chirurgien et son aide, une surveillance non garantie qui sont des facteurs compromettant la prise du lambeau.

Dans cette étude, La moyenne d'âge était de 34 ans avec des âges inférieur et supérieur de 02 ans et 65 ans. Le sexe féminin était prédominant dans 59\% des cas avec un sex ratio de 0,68 et la tranche d'âge de 31-45 ans était la plus touchée dans $31,25 \%$. L'étiologie tumorale était la plus fréquente dans $43,75 \%$ des cas suivi des PDS post- traumatique dans $34,38 \%$.

L'âge moyen varie de 30 à 33,4 ans avec des extrêmes de 4 et 76 ans et une prédominance masculine observée (Anzouan K E et al., 2015 ; Agbara $\mathrm{R}$ et al., 2016). Le traumatisme dans $58,4 \%$ des cas, était le facteur étiologique le plus courant, suivi de l'exérèse tumorale dans 23,4\% (Agbara R et al., 2016). la pathologie infectieuse, notamment les séquelles de noma est la plus fréquente dans $45,34 \%$ des cas suivi de l'exérèse tumorale dans 33,33\% ( Anzouan K E et al., 2015).

La PDS de la région parotidienne représentait la partie la plus atteinte dans $21,88 \%$ des cas et était secondaire à une exérèse tumorale et à un traumatisme par accident de la voie publique. Le choix d'un lambeau à utiliser pour une PDS, est fonction du siège, de l'unité esthétique, de la fonction compromise, de l'expérience du chirurgien, et de la morbidité du site donneur. Les lambeaux peuvent être taillé au hasard, pédicule ou libre, et ce, de façon local, régional (ou locorégional) ou à distance. Dans notre étude, Les lambeaux locaux : Ginestet, Eslander, Karapandzick, nasogénien et Webster, ont été utiliser pour les PDS des lèvres et de l'aile narinaire. Les lambeaux 
régionaux : frontal, du scalp, sous mental, du sternocléidomastoïdien, du temporal, fascio-cutané et cutané ont été utilisés pour les PDS de la région parotidienne, jugale, en bouche, de l'aile narinaire, de l'hémiface, du front et du cuir chevelu. Les lambeaux à distance : pédiculé (grand dorsal, grand pectoral) et libre (antébrachial, et de fibula+palette) ont été utilisé dans les PDS importantes emportant souvent l'os après exérèse tumorale ou dont l'arc de rotation des lambeaux locorégionaux ne permet pas de compenser la PDS. Généralement, les PDS de la joue peuvent être reconstruits en utilisant une fermeture primaire, une greffe de peau pleine épaisseur, un lambeau rhomboïde, un lambeau bilobé, un lambeau d'avancement cervico-facial, un lambeau de fascia temporopariétal, un lambeau de muscle temporal, un lambeau sous-mental, un lambeau deltopectoral, un lambeau frontal, un avantbras radial libre lambeau et lambeau antérolatéral de cuisse libre. Les PDS de la lèvre peuvent être reconstruits à l'aide de la fermeture primaire, d'un d'Abbe, d'Abbe- Estlander, d'Estlander, de Gillis, de Karapandzic, d'avancement de Webster, de Webster-Bernard, de marche, de Schuchardt, nasolabial, de Fujimori, d'avant-bras radial libre total et latéral de bras (Pletcher SD et al., 2005 ; Pellini R et al., 2006). De nos jours, la méthode la plus populaire pour la prise en charge des PDS dans la région de la tête et du cou est représentée par la transposition de lambeaux microvasculaires libres. L'introduction de lambeaux libres en chirurgie reconstructive a fourni au chirurgien de la tête et du cou une grande variété de tissus disponibles, tels que la peau, les muscles et les os, pour une restauration optimale de la forme et de la fonction (Varvares MA et al., 2005 ; Beausang ES et al., 2003). Cependant, tous les patients ne sont pas des candidats appropriés pour une reconstruction par lambeau libre. De plus, toutes les PDS ne nécessitent pas un transfert libre pour obtenir de bons résultats fonctionnels (Mahieu $\mathrm{R}$ et al., 2016). La nécrose partielle était la complication la plus fréquente rencontrée dans notre étude et se retrouve dans les lambeaux locaux taillés au hasard et fasciocutané régionaux.

\section{Conclusion}

Les pertes de substance maxillo-faciale sont souvent responsables de certains préjudice fonctionnel et esthétique qui peuvent altérer la personnalité. La reconstruction de ces PDS en constitue un véritable défi pour le chirurgien cervico-maxillo-faciale et/ou plasticien afin de rétablir la fonction compromise ou de donner un visage socialement acceptable et reconnaissable. Cette reconstruction fait appel à des lambeaux locorégionaux et à distance avec des indications bien précises. La maitrise de l'une ou l'autre technique dépends de l'expérience du chirurgien, de la cause de la PDS, et des antécédents des patients. 


\section{References:}

1. Agbara R, Obiadazie AC, Fomete B, Omeje KU. Orofacial Soft Tissue Reconstruction with Locoregional Flaps in a Health ResourceDepleted Environment: Experiences from Nigeria. Arch Plast Surg. 2016;43(3):265-71.

2. Anzouan K E, Assouan C, Nguessan D, Vieira S, Salami A, Konan E, Assa A. Utilisation des lambeaux dans les pertes de substance de la face au CHU de Treichville. Rev Col Odonto-Stomatol Afr Chir Maxillo-fac, 2015 Vol 22, n 1 , pp. 41-45

3. Baliarsing AS, Thorat TS, Gupta A. Flap selection in head and neck cancer reconstruction. Int J Otorhinolaryngol Clin 2013; 5:63-76

4. Beausang ES, Ang EE, Lipa JE, Irish JC, Brown DH, Gullane PJ, Neligan PC. Microvascular free tissue transfer in elderly patients: the Toronto experience. Head Neck. 2003 Jul;25(7):549-53.

5. Deganello A. Modern oral cavity reconstruction with free flaps and pedicled flaps. J Aesth Reconstr Surg 2015;1:4.

6. Mahieu R, Colletti G, Bonomo P, Parrinello G, Iavarone A, Dolivet G, Livi L, Deganello A. Head and neck reconstruction with pedicled flaps in the free flap era. Acta Otorhinolaryngol Ital. 2016 Dec;36(6):459468.

7. Pellini R, Pichi B, Marchesi P, et al. External monitor for buried free flaps in head and neck reconstructions. Acta Otorhinolaryngol Ital 2006;26:1-6.

8. Pletcher SD, Kim DW. Current concepts in cheek reconstruction. Facial Plast Surg Clin North Am 2005;13:267-81.

9. Squaquara R, Kim Evans KF, Spanio di Spilimbergo S, Mardini S. Intraoral reconstruction using local and regional flaps. Semin Plast Surg. 2010;24(2):198-211.

10. Varvares MA, Lin D, Hadlock T, et al. Success of multiple, sequential, free tissue transfers to the head and neck. Laryn- goscope 2005;115:101-4.

11. Zwetyenga N, Lutz J-C, Vidal N, El-Bouihi M, Siberchicot F, Martin D. Le lambeau sous-mental pédiculé. Rev Stomatol Chir Maxillofac 2007;108:210-214 